\title{
The Clinical Value of the RA-Adjusted Fracture Risk Assessment Tool in the Fracture Risk Prediction of Patients with Type 2 Diabetes Mellitus in China
}

Ling $\mathrm{Hu}^{*}$

Ting Li*

Yi Zou

Xiao-Ling Yin

Hui Gan

Department of Endocrinology, The Third Affiliated Hospital of Nanchang University, Nanchang 330000, People's Republic of China

*These authors contributed equally to this work
This article was published in the following Dove Press journal:

International Journal of General Medicine

\begin{abstract}
Objective: This study aimed to explore the clinical value of the fracture risk assessment tool (FRAX) in the fracture risk prediction of Chinese patients after replacing rheumatoid arthritis (RA) with type 2 diabetes mellitus (T2DM) in the FRAX algorithm.

Methods: A total of 1,047 patients with T2DM from the Endocrinology Department of the Third Affiliated Hospital of Nanchang University were enrolled in this study. Dual-energy X-ray absorptiometry (DXA) was then used to detect their bone density. RA in the FRAX algorithm was replaced with T2DM, and the new RA-adjusted FRAX was used to assess the fracture risk of the patients.

Results: The sensitivity, specificity, and Youden's index of the RA-adjusted FRAX to the treatment opinions on T2DM-associated hip fractures were 0.4761, 0.9642, and 0.4403, respectively, while the sensitivity, specificity, and Youden's index of RA-adjusted FRAX to the treatment opinions on T2DM-associated major bone osteoporotic fractures were 0.0080 , $1.0000,0.0080$, respectively. The DXA and RA-adjusted FRAX both showed acceptable consistency in the treatment recommendations for hip fractures in patients with T2DM $(\kappa=$ 0.49 ) but had poor consistency in treatment recommendations for major bone osteoporotic fractures $(\kappa=0.010)$. The body mass index (BMI) scores, femoral neck-bone mineral densities, and number of males in the same treatment opinion group were significantly higher than in the different treatment opinions group $(P<0.001)$.

Conclusion: RA-adjusted FRAX is a useful clinical tool for evaluation of hip fracture risk for Chinese patients with T2DM, and the accuracy of fracture risk prediction for male patients with T2DM and patients with T2DM with high BMI scores or high femoral neckbone mineral density is higher.
\end{abstract}

Keywords: type 2 diabetes mellitus, FRAX, fracture risk, bone mineral density, osteoporosis

\section{Introduction}

The number of diabetic patients has continued to increase in recent years: worldwide, there were 371 million patients with diabetes in 2012, which increased to 382 million in $2013 .^{1-4}$ According to the results of mathematical modeling, there will be 552 million people with diabetes worldwide by $2030 .^{2}$

Osteoporosis (OP) is a common bone disease characterized by decreased bone mass and the destruction of bone microstructure, which leads to decreased bone strength, increased fragility, and increased risk of fractures, further causing pain,
Correspondence: Ling Hu

Department of Endocrinology, The Third Affiliated Hospital of Nanchang University

No. 128 of Xiangshan North Road, Donghu District, Nanchang 330000, People's

Republic of China

$\mathrm{Tel}+86$ I3970075 II9

Email drhu_hlII72@I63.com 
impaired function, a decline in quality of life, and death. ${ }^{5}$ One of the most serious consequences of OP is fracture, with the most common site of fracture being the thoracolumbar vertebrae, hips, and forearms.

Many studies have revealed a correlation between diabetes and OP and found that the risk of fracture in diabetic patients is significantly increased. ${ }^{6-10}$ The bone quality of patients with T2DM is poorer and the risk of osteoporotic fracture therefore significantly higher than that of nondiabetic patients. ${ }^{11-15}$ Therefore, early identification of high fracture risk in these patients, and the administration of anti-OP treatment, can significantly reduce their fracture risk.

In current clinical practice, the standard diagnostic technique for assessing OP, predicting fracture risk, and monitoring treatment is measuring bone mineral density (BMD) using dual-energy X-ray absorptiometry (DXA). However, patients with T2DM often have an increased risk of osteoporotic fractures even when they have a normal or higher BMD. ${ }^{7,9,16-20}$ Therefore, BMD cannot always reliably indicate the fracture risk of patients with T2DM. The fracture risk assessment tool (FRAX) is widely used to assess the 10-year probability of major fracture (PMF) or hip osteoporotic fractures and the 10year probability of major osteoporotic fractures (PMOF; such fractures include the clinical spine, forearms, hips, or shoulders). It is undertaken using computer software that combines the probability of fracture with a variety of clinical risk factors and the BMD of the femoral neck. However, many studies have found that FRAX can underestimate the risk of fracture in patients with T2DM. ${ }^{21}$ The purpose of the present study is to evaluate patients with T2DM using FRAX with RA as the equivalent variable of T2DM and to correct the predictive value of fracture risk in patients with T2DM, thereby exploring the clinical value of RA-adjusted FRAX as a fracture risk prediction tool in the risk assessment of T2DM-associated osteoporotic fractures.

\section{Subjects and Methods Subjects}

A total of 1,047 patients with T2DM who were hospitalized in the Endocrinology Department of the Third Affiliated Hospital of Nanchang University between January 2019 and December 2019 were enrolled.

Inclusion criteria: (1) Patient met the diagnostic criteria for T2DM established by the World Health Organization
(WHO) in 1999; (2) aged 40-90; (3) patient was aware of the research and voluntarily cooperated.

Exclusion criteria: (1) Patient had type 1 diabetes mellitus (T1DM) or other types of diabetes; (2) patient had recently used drugs that affect bone metabolism; (3) patient had severe heart, lung, liver, or kidney disease; (4) patient had malignant tumors; (5) patient was pregnant.

\section{Methods}

A standardized questionnaire was used to collect and record the name, gender, age, lifestyle habits (current smoking behavior, daily alcohol intake, etc.), past history (past history of fractures, history of rheumatoid arthritis, secondary osteoporosis, cirrhosis, etc.), medication (long-term use of glucocorticoid drugs, etc.), family history (parental hip fracture history, etc.). The height, weight, and body mass index (BMI) of each patient were measured according to uniform standards. BMI $=$ weight $/$ height $^{2}$ (unit: $\mathrm{kg} / \mathrm{m}^{2}$ ).

Glycated hemoglobin (HbA1c) and BMD detection: A high-performance liquid chromatography (HPLC) ionexchange method (Huizhong MQ-2000PT) was used to determine the level of HbA1c. Professionally trained fixed operators used DXA (Hologic Explorer, USA) to perform scans on the patients. The BMD and $\mathrm{T}$ value of the spine and femur were obtained from the test data. The patients were classified according to the 1995 WHO diagnostic criteria for $\mathrm{OP}$ : $\mathrm{OP}=\mathrm{T} \leq 2.5$; bone loss $=-2.5<$ $\mathrm{T}<-1$; normal bone mass $=\mathrm{T} \geq-1$. For premenopausal women and men under the age of 50, BMD was determined by the $\mathrm{Z}$-value of the same race.

FRAX index calculation: The FRAX online platform was accessed and the Asian-China Assessment System used to calculate the probability of hip fracture and any major osteoporotic fracture in the next 10 years. The risk factors in the FRAX algorithm include age, gender, height, weight, history of previous fractures, parents' history of hip fractures, current smoking behavior, long-term use of corticosteroids, history of RA, daily alcohol intake of 3 units or more, secondary OP, and femoral neck-bone density. For the present study, the history of RA was replaced with T2DM in the algorithm. Using the 10-year probability of hip fracture $\geq 3 \%$ or the 10 -year probability of any major osteoporotic fracture $\geq 20 \%$ as the treatment threshold, the treatment recommendations were calculated. According to the FRAX-RA evaluation results, it is recommended to start anti-osteoporosis therapy if the probability of hip fracture within 10 years is $\geq 3 \%$ and the patient is at high risk of fracture. On the contrary, anti- 
osteoporosis therapy is not recommended for patients with low fracture risk. If the probability of osteoporotic fracture at the main site is $\geq 20 \%$ within 10 years, anti-osteoporosis therapy is recommended for patients with high fracture risk; otherwise, anti-osteoporosis therapy is not recommended for patients with low fracture risk.

\section{Statistical Analysis}

The data were processed using SPSS 22.0. $P<0.05$ was considered statistically significant. The measurement data were expressed as mean \pm standard deviation $(x \pm \mathrm{SD})$. All non-normally distributed parameters were logarithmically converted before statistical analysis. Count data were compared using an $X^{2}$ test. Measurement data were evaluated using a $t$-test or analysis of variance (ANOVA). The evaluation index of diagnostic ability included sensitivity, specificity, and Youden's index. The consistency analysis was performed using the $\kappa$ test: $\kappa<0.4$ indicated poor consistency, $0.4 \leq \kappa \leq 0.7$ indicated medium consistency, and $\kappa>0.7$ indicated good consistency.

\section{Results}

\section{General Patient Data}

A total of 1,047 patients with T2DM were included in this study. Among these, 592 were male and 455 were female. Table 1 shows the general clinical characteristics of the patients.

Of the total number of patients, 376 had OP (35.91\%), 494 had low BMD (47.18\%), and 177 had normal BMD (16.91\%). The number of females was significantly higher in the OP group than in the low BMD group and normal BMD group $(P<0.05)$. However, there was no significant difference between the low BMD group and normal BMD group in the ratio of males to females $(P>0.05)$. There were significant differences in age, weight, height, and BMI score between the three groups $(P<0.05)$, and it was found that
BMD decreased as age increased and weight, height, and BMI score decreased. No significant difference was found between the groups in $\mathrm{HbA1c}(P>0.05)$.

\section{Evaluating the Sensitivity, Specificity, and Youden's Index of Anti-OP Treatment in Patients with T2DM Using RA-Adjusted FRAX}

In this study, 376 patients had OP and 671 patients had either low or normal BMD. The RA-adjusted FRAX predicted that, for major sites, three patients had high fracture risk and 1,044 had a lower fracture risk, while for the hip, 203 patients were predicted to have high fracture risk and 844 patients to have a lower fracture risk (Table 2).

Table 3 shows the sensitivity, specificity, positive predictive value, negative predictive value, and Youden's index of RA-adjusted FRAX in evaluating the initiation of anti-OP therapy in patients with T2DM using DXA as a reference. Consistency analysis revealed that, when the treatment threshold was defined as the probability of hip fracture $\geq 3 \%$ within 10 years, it was consistent with the DXA results $(\kappa=0.49, P<0.001)$ and the consistency was acceptable; when the treatment threshold was defined as the probability of any major osteoporotic fracture $>20 \%$, it was consistent with the DXA results $(\kappa=0.010, P<$ 0.001 ) but the consistency was poor.

\section{Comparison of Basic Data Between the Two Groups}

The patients were divided into two groups based on the results of the DXA and RA-adjusted FRAX hip fracture risk assessment: a same treatment opinion group (both DXA and RAadjusted FRAX suggested starting anti-OP treatment or not) and a different treatment opinions group (DXA and RAadjusted FRAX differed on whether to start anti-OP treatment

Table I General Clinical Characteristics of T2DM Patients $(X \pm S D)$

\begin{tabular}{|l|l|l|l|}
\hline Index & Osteoporosis (n=376) & Low BMD (n=494) & Normal BMD (n=I77) \\
\hline Gender(male/female) & $135 / 241^{\mathrm{bc}}$ & $337 / 157^{\mathrm{e}}$ & $120 / 57$ \\
Age(year) & $64.35 \pm 8.99^{\mathrm{bc}}$ & $59.96 \pm 9.04^{\mathrm{b}}$ & $55.77 \pm 9.92$ \\
Weight $(\mathrm{Kg})$ & $59.01 \pm 9.36^{\mathrm{bc}}$ & $66.91 \pm 10.57^{\mathrm{b}}$ & $71.55 \pm 11.89$ \\
Height $(\mathrm{cm})$ & $156.63 \pm 8.03^{\mathrm{bc}}$ & $162.84 \pm 8.04^{\mathrm{d}}$ & $164.38 \pm 7.94$ \\
BMI( $\left(\mathrm{kg} / \mathrm{m}^{2}\right)$ & $24.04 \pm 3.27^{\mathrm{bc}}$ & $25.18 \pm 3.25^{\mathrm{b}}$ & $26.39 \pm 3.28$ \\
$\mathrm{BMD}^{\mathrm{a}}\left(\mathrm{g} / \mathrm{cm}^{2}\right)$ & $0.58 \pm 0.09^{\mathrm{c}}$ & $0.72 \pm 0.08^{\mathrm{b}}$ & $0.85 \pm 0.09$ \\
HbAIC(\%) & $9.19 \pm 2.59^{\mathrm{ef}}$ & $8.96 \pm 2.34^{\mathrm{e}}$ & $9.06 \pm 2.42$ \\
\hline
\end{tabular}

Notes: ${ }^{\mathrm{a}} \mathrm{Femoral}$ neck bone density; ${ }^{\mathrm{b} C}$ Compared with the normal bone density group, $\mathrm{P}<0.00 \mathrm{I} ;{ }^{\mathrm{c} C}$ Compared with the low bone density group, $\mathrm{P}<0.00 \mathrm{I}$; ${ }^{\mathrm{d} C} \mathrm{Compared}$ with the normal BMD group, $\mathrm{P}<0.05$; ${ }^{\mathrm{e} C o m p a r e d}$ with the normal BMD group, $\mathrm{P}>0.05$; ${ }^{\mathrm{f} C o m p a r e d}$ with the low BMD group, $\mathrm{P}>0.05$. 
Table 2 Analysis of Fracture Risk Assessment Results of RA-Adjusted FRAX and DXA in Patients with T2DM (Cases)

\begin{tabular}{|l|l|l|l|}
\hline \multirow{2}{*}{ Project } & \multicolumn{2}{l|}{ DXA } & Low Bone Density, Normal Bone Density(n) \\
\cline { 2 - 4 } & Osteoporosis (n) & & Total(n) \\
\hline RA-adjusted FRAX (PMOF) & & 0 & 3 \\
High fracture risk (n) & 3 & 671 & 1044 \\
Low fracture risk (n) & 373 & 671 & 1047 \\
Total (n) & 376 & & 203 \\
RA-adjusted FRAX (PHF) & & 24 & 844 \\
High fracture risk (n) & 179 & 647 & 1047 \\
Low fracture risk (n) & 197 & 671 & \\
Total (n) & 376 & & \\
\hline
\end{tabular}

Table 3 Diagnostic Ability of RA-Adjusted FRAX in T2DM Patients Who Need to Initiate Anti-Osteoporosis Therapy

\begin{tabular}{|l|l|l|}
\hline & RA-Adjusted FRAX(PMOF) & RA-Adjusted FRAX(PHF) \\
\hline Sensitivity & 0.0080 & 0.4761 \\
Specificity & 1.0000 & 0.9642 \\
Positive predictive value & 1.0000 & 0.8818 \\
Negative predictive value & 0.6427 & 0.7666 \\
Youden index & 0.0080 & 0.4403 \\
\hline
\end{tabular}

or not). Table 4 shows that there was a significant difference in gender between the same treatment opinion group and the different treatment opinions group $(P<0.05)$, and the number of males was significantly higher in the same treatment opinion group than in the different treatment opinions group. BMI scores and femoral neck BMDs were significantly higher in the same treatment opinion group than in the different treatment opinions group $(P<0.05)$. However, there were no significant differences in age and $\mathrm{HbAlc}$ between the same treatment opinion group and the different treatment opinions group $(P>0.05)$.

\section{Discussion}

T2DM and OP are the two most common metabolic endocrine diseases in the middle-aged and elderly, and their prevalence increases gradually with age. Patients with
T2DM have an increased risk of fracture, especially hip fractures, and their RR values range from 1.2 to $1.7 .^{14,22-26}$ However, the underlying mechanism of the increased risk of fracture in patients with T2DM with normal or higher BMD remains unclear. $^{27,28}$ Some current studies have concluded that non-enzyme-promoting glycosylation of T2DM leads to the accumulation of advanced glycation end-products (AGE) in the organic bone matrix, decreasing bone strength and increasing bone fragility. ${ }^{29-32}$ In middle-aged and elderly people, the level of estrogen is decreased, the intestinal absorption of calcium is decreased, 1, 25- $(\mathrm{OH})_{2}$, and $\mathrm{D}_{3}$ production in the kidney are decreased, and secondary hyperparathyroidism occurs and promotes bone resorption. ${ }^{33}$ In patients with T2DM, adipose tissue, especially visceral fat, may have a negative impact on bone health by secreting adipokines and

Table 4 Clinical Data of T2DM Patients Provided by RA-Adjusted FRAX and DXA in the Same Treatment Opinion Group and the Different Treatment Opinions Group

\begin{tabular}{|l|l|l|l|}
\hline & $\begin{array}{l}\text { The Same Treatment Opinion Group } \\
\mathbf{n = 8 2 6}\end{array}$ & $\begin{array}{l}\text { The Different Treatment Opinions Group } \\
\mathbf{n = 2 2 1}\end{array}$ & P value \\
\hline Age(year) & $60.88 \pm 9.90$ & $60.66 \pm 8.66$ & 0.746 \\
Gender(male/female) & $491 / 335$ & $101 / 120$ & $<0.001$ \\
BMI $\left(\mathrm{kg} / \mathrm{m}^{2}\right)$ & $25.21 \pm 3.32$ & $24.08 \pm 3.36$ & $<0.001$ \\
HbAIC $(\%)$ & $9.03 \pm 2.39$ & $9.18 \pm 2.67$ & 0.462 \\
Femoral neck BMD $\left(\mathrm{g} / \mathrm{cm}^{2}\right)$ & $0.71 \pm 0.13$ & $0.63 \pm 0.06$ & $<0.001$ \\
\hline
\end{tabular}


inflammatory factors that increase bone resorption. ${ }^{19}$ The accumulation of bone-marrow fat may also increase the risk of fracture in these patients. ${ }^{7,34}$

As a fracture risk assessment tool recommended by WHO, FRAX is economical, convenient, and easy to operate. Using this tool, individual fracture risk can be assessed according to clinically available risk factors with or without BMD, providing a way for clinicians to identify the high risk of fracture early and make appropriate treatment decisions. However, it has some limitations: FRAX is only suitable for people not undergoing anti-OP treatment, and it does not include many other risk factors, such as dosage and course of glucocorticoid application and the etiology and type of secondary OP or diabetes mellitus. In the present study, only the previous fracture history was considered, and the frequency, type, and severity of previous fractures were not included.

T2DM, independent of other risk factors, significantly increases the risk of fracture; however, the clinical risk factors included in the FRAX algorithm do not include T2DM. In response to this, Schacter et $\mathrm{al}^{35}$ and Leslie et $\mathrm{al}^{36}$ made some suggestions for improving FRAX's prediction of fracture risk in patients with T2DM, such as the equivalent replacement of RA with T2DM in the algorithm, increasing the trabecular bone score (TBS) value of the lumbar spine, decreasing the $\mathrm{T}$ value of the femoral neck by $0.5 \mathrm{SD}$, and increasing the age by 10 years. Leslie et $\mathrm{al}^{36}$ found that implementing all these suggestions was better than the unadjusted FRAX score but implementing none of them was also better. As such, these adjustments can improve FRAX's assessment of fracture risk in patients with T2DM to a certain extent. In the present study, to improve the accuracy of FRAX in the fracture risk assessment of T2DM patients, RA was chosen as the equivalent variable of T2DM based on the Chinese expert consensus on the fracture risk management of diabetic patients. ${ }^{37}$ However, the specific clinical predictive value of RA-adjusted FRAX in patients with T2DM remains unclear.

The present study found that BMD decreases as age increases and BMI score decreases and that the incidence of OP in women is significantly higher than in men. This is consistent with previous studies. ${ }^{19,38-41}$ A number of studies have also suggested that there is a negative correlation between BMD and HbAlc in patients with T2DM, ${ }^{42-44}$ but the present study found no significant difference in HbA1C level between the groups.

In this study, it was concluded that RA-adjusted FRAX with a 10 -year hip fracture probability of $\geq 3 \%$ as the threshold was more consistent with DXA than with a major osteoporosis-fracture probability of $\geq 20 \%$ as the threshold. It can be considered that a RA-adjusted FRAX threshold with a hip fracture risk of $\geq 3 \%$ is of higher diagnostic value, while a RA-adjusted FRAX threshold with a probability of $\geq 20 \%$ of any major osteoporosis-related fracture may lead to missed diagnosis of osteoporosis in type 2 diabetes patients and delay their treatment. However, the sensitivity of RA-adjusted FRAX with hip fracture risk $\geq 3 \%$ as a treatment threshold is still not satisfactory. Further research and analysis are needed to determine the intervention threshold of RA-adjusted FRAX in type 2 diabetes mellitus (T2DM) population in China.

The present study also found significant differences between the same treatment opinion group and the different treatment opinions group in gender, BMI score, and BMD of the femoral neck $(P<0.05)$, although there were no significant differences in age and $\mathrm{HbA} 1 \mathrm{C}$ between the two groups $(P>0.05)$. The BMI score, femoral neck $\mathrm{BMD}$, and number of males were significantly higher in the same treatment opinion group than in the different treatment opinions group. This suggests that RA-adjusted FRAX is more clinically accurate in assessing the risk of hip fracture in males, those with higher BMI, and those with higher femoral neck BMD in T2DM.

\section{Conclusion}

After replacing diabetes with RA in the FRAX algorithm, the tool demonstrated a good ability to assess the risk of hip fracture in patients with T2DM. The accuracy of fracture risk prediction for male patients with T2DM and patients with T2DM with high BMI scores or high femoral neckbone mineral density was also higher. Although there is no FRAX intervention threshold for T2DM in China, using the 10 -year probability of hip fracture risk $\geq 3 \%$ as the intervention threshold value offers greater clinical value.

\section{Ethics Approval and Consent to Participate}

I confirm that I have read the Editorial Policy pages. This study was conducted with approval from the Ethics Committee of The third Affiliated Hospital of Nanchang University. This study was conducted in accordance with the declaration of Helsinki. Written informed consent was obtained from all participants.

\section{Consent for Publication}

All participants signed a document of informed consent. 


\section{Acknowledgments}

We would like to acknowledge the hard and dedicated work of all the staff that implemented the intervention and evaluation components of the study.

\section{Disclosure}

The authors declare that they have no competing interests.

\section{References}

1. Kulwas A, Drela E, Jundziłł W, Góralczyk B, Ruszkowska-Ciastek B, Rość D. Circulating endothelial progenitor cells and angiogenic factors in diabetes complicated diabetic foot and without foot complications. J Diabetes Complications. 2015;29(5):686-690. doi:10.1016/j.jdiacomp.2015.03.013

2. Waniczek D, Kozowicz A, Muc-Wierzgoń M, Kokot T, Świętochowska E, Nowakowska-Zajdel E. Adjunct methods of the standard diabetic foot ulceration therapy. Evid Based Complement Alternat Med. 2013;2013:1-12. doi:10.1155/2013/243568

3. Nguyen TT, Ding D, Wolter WR, et al. Validation of matrix metalloproteinase-9 (MMP-9) as a novel target for treatment of diabetic foot ulcers in humans and discovery of a potent and selective small-molecule MMP-9 inhibitor that accelerates healing. $J$ Med Chem. 2018;61(19):8825-8837. doi:10.1021/acs.jmedchem.8b01005

4. Guariguata L, Whiting D, Hambleton I, Beagley J, Linnenkamp U, Shaw J. Global estimates of diabetes prevalence for 2013 and projections for 2035. Diabetes Res Clin Pract. 2014;103(2):137-149. doi:10.1016/j.diabres.2013.11.002

5. Siris E, Adler R, Bilezikian J, et al. The clinical diagnosis of osteoporosis: a position statement from the National Bone Health Alliance Working Group. Osteoporos Int. 2014;25(5):1439-1443. doi:10.1007/s00198-014-2655-z

6. Jiang N, Xia W. Assessment of bone quality in patients with diabetes mellitus. Osteoporos Int. 2018;29(8):1721-1736. doi:10.1007/ s00198-018-4532-7

7. Poiana C, Capatina C. Fracture risk assessment in patients with diabetes mellitus. $J$ Clin Densitom. 2017;20(3):432-443. doi:10.1016/j.jocd.2017.06.011

8. Thong E, Herath M, Weber D, et al. Fracture risk in young and middle-aged adults with type 1 diabetes mellitus: a systematic review and meta-analysis. Clin Endocrinol (Oxf). 2018;89(3):314-323. doi:10.1111/cen.13761

9. Wang H, Ba Y, Xing Q, Du J. Diabetes mellitus and the risk of fractures at specific sites: a meta-analysis. BMJ Open. 2019;9(1): e024067. doi:10.1136/bmjopen-2018-024067

10. Sato M, Ye W, Sugihara T, Isaka Y. Fracture risk and healthcare resource utilization and costs among osteoporosis patients with type 2 diabetes mellitus and without diabetes mellitus in Japan: retrospective analysis of a hospital claims database. BMC Musculoskelet Disord. 2016;17(1):489. doi:10.1186/s12891-016-1344-9

11. Tebé C, Martinez-Laguna D, Moreno V, et al. Differential mortality and the excess rates of hip fracture associated with type 2 diabetes: accounting for competing risks in fracture prediction matters. $J$ Bone Miner Res. 2018;33(8):1417-1421. doi:10.1002/jbmr.3435

12. Vestergaard P. Discrepancies in bone mineral density and fracture risk in patients with type 1 and type 2 diabetes-a meta-analysis. Osteoporos Int. 2007;18(4):427-444. doi:10.1007/s00198-006-0253-4

13. Gilbert MP, Pratley RE. The impact of diabetes and diabetes medications on bone health. Endocr Rev. 2015;36(2):194-213. doi:10.1210/ er.2012-1042
14. Fan Y, Wei F, Lang Y, Liu Y. Diabetes mellitus and risk of hip fractures: a meta-analysis. Osteoporos Int. 2016;27(1):219-228. doi:10.1007/s00198-015-3279-7

15. Majumdar SR, Leslie WD, Lix LM, et al. Longer duration of diabetes strongly impacts fracture risk assessment: the manitoba BMD cohort. $J$ Clin Endocrinol Metab. 2016;101(11):4489-4496. doi:10.1210/ jc.2016-2569

16. Liu M, Lu Y, Cheng X, et al. Relationship between abnormal glucose metabolism and osteoporosis in Han Chinese men over the age of 50 years. Clin Interv Aging. 2019;14:445-451. doi:10.2147/CIA. S164021

17. Schwartz A, Vittinghoff E, Bauer D, et al. Association of BMD and FRAX score with risk of fracture in older adults with type 2 diabetes. JAMA. 2011;305(21):2184-2192. doi:10.1001/jama.2011.715

18. Guo Y, Wang Y, Chen F, Wang J, Wang D. Assessment of risk factors for fractures in patients with type 2 diabetes over 60 years old: a cross-sectional study from Northeast China. $J$ Diabetes Res. 2020;2020:1508258. doi:10.1155/2020/1508258

19. Palermo A, Tuccinardi D, Defeudis G, et al. BMI and BMD: the potential interplay between obesity and bone fragility. Int $J$ Environ Res Public Health. 2016;13(6):544. doi:10.3390/ijerph13060544

20. Schwartz A. Epidemiology of fractures in type 2 diabetes. Bone. 2016;82:2-8. doi:10.1016/j.bone.2015.05.032

21. Giangregorio L, Leslie W, Lix L, et al. FRAX underestimates fracture risk in patients with diabetes. Bone Miner Res. 2012;27(2):301-308. doi: $10.1002 / \mathrm{jbmr} .556$

22. Bai J, Gao Q, Wang C, Dai J. Diabetes mellitus and risk of low-energy fracture: a meta-analysis. Aging Clin Exp Res. 2020;32 (11):2173-2186. doi:10.1007/s40520-019-01417-x

23. Jia $\mathrm{P}, \mathrm{Bao} \mathrm{L}, \mathrm{Chen} \mathrm{H}$, et al. Risk of low-energy fracture in type 2 diabetes patients: a meta-analysis of observational studies. Osteoporos Int. 2017;28(11):3113-3121. doi:10.1007/s00198-017-4183-0

24. Rubin MR. Skeletal fragility in diabetes. Ann N Y Acad Sci. 2017;1402(1):18-30. doi:10.1111/nyas. 13463

25. Moayeri A, Mohamadpour M, Mousavi SF, Shirzadpour E, Mohamadpour S, Amraei M. Fracture risk in patients with type 2 diabetes mellitus and possible risk factors: a systematic review and meta-analysis. Ther Clin Risk Manag. 2017;13:455-468. doi:10.2147/TCRM.S131945

26. Hamilton E, Davis WA, Bruce DG, Davis TM. Influence of premature mortality on the link between type 2 diabetes and hip fracture: the fremantle diabetes study. J Clin Endocrinol Metab. 2017;102 (2):551-559. doi:10.1210/jc.2016-3570

27. Napoli N, Chandran M, Pierroz D, Abrahamsen B, Schwartz A, Ferrari S. Mechanisms of diabetes mellitus-induced bone fragility. Nat Rev Endocrinol. 2017;13(4):208-219. doi:10.1038/ nrendo.2016.153

28. Conte C, Epstein S, Napoli N. Insulin resistance and bone: a biological partnership. Acta Diabetol. 2018;55(4):305-314. doi:10.1007/s00592-018-1101-7

29. Yamagishi S, Nakamura K, Inoue H. Possible participation of advanced glycation end products in the pathogenesis of osteoporosis in diabetic patients. Med Hypotheses. 2005;65(6):1013-1015. doi:10.1016/j.mehy.2005.07.017

30. Karim L, Moulton J, Van Vliet M, et al. Bone microarchitecture, biomechanical properties, and advanced glycation end-products in the proximal femur of adults with type 2 diabetes. Bone. 2018;114:32-39. doi:10.1016/j.bone.2018.05.030

31. Garnero P, Borel O, Gineyts E, et al. Extracellular post-translational modifications of collagen are major determinants of biomechanical properties of fetal bovine cortical bone. Bone. 2006;38(3):300-309. doi:10.1016/j.bone.2005.09.014

32. Alikhani M, Alikhani Z, Boyd C, et al. Advanced glycation end products stimulate osteoblast apoptosis via the MAP kinase and cytosolic apoptotic pathways. Bone. 2007;40(2):345-353. doi:10.1016/j.bone.2006.09.011 
33. Wang X, Yu J, Wang X, et al. The associations between hypovitaminosis $\mathrm{d}$, higher pth levels with bone mineral densities, and risk of the 10-year probability of major osteoporotic fractures in chinese patients with t2dm. Endocr Pract. 2018;24(4):334-341. doi:10.4158/EP2017-0164

34. Patsch J, Li X, Baum T, et al. Bone marrow fat composition as a novel imaging biomarker in postmenopausal women with prevalen fragility fractures. $J$ Bone Miner Res. 2013;28(8):1721-1728. doi: $10.1002 /$ jbmr. 1950

35. Schacter G, Leslie W. DXA-based measurements in diabetes: can they predict fracture risk? Calcif Tissue Int. 2017;100(2):150-164. doi:10.1007/s00223-016-0191-x

36. Leslie WD, Johansson H, McCloskey EV, Harvey NC, Kanis JA, Hans D. Comparison of methods for improving fracture risk assessment in diabetes: the manitoba BMD registry. $J$ Bone Miner Res. 2018;33(11):1923-1930. doi:10.1002/jbmr.3538

37. Chinese Society of Osteoporosis and Bone Mineral Research; Chinese Society of Endocrinology; Chinese Diabetes Society; Association; C. M.; Chinese Endocrinologist Association; Association, C. M. D. Chinese expert consensus on the management of fracture risk in diabetic patients. Chin J Osteoporos Bone Miner Res. 2019;12(4):319-335.

38. Chen P, Li Z, Hu Y. Prevalence of osteoporosis in China: a meta-analysis and systematic review. BMC Public Health. 2016;16 (1):1039. doi:10.1186/s12889-016-3712-7
39. Tian L, Yang R, Wei L, et al. Prevalence of osteoporosis and related lifestyle and metabolic factors of postmenopausal women and elderly men: a cross-sectional study in Gansu province, Northwestern of China. Medicine. 2017;96(43):e8294. doi:10.1097/MD.00000000 00008294

40. Cui L, Chen L, Xia W, et al. Vertebral fracture in postmenopausal Chinese women: a population-based study. Osteoporos Int. 2017;28 (9):2583-2590. doi:10.1007/s00198-017-4085-1

41. Jepsen K, Schlecht S. Biomechanical mechanisms: resolving the apparent conundrum of why individuals with type II diabetes show increased fracture incidence despite having normal BMD. $J$ Bone Miner Res. 2014;29(4):784-786. doi:10.1002/jbmr.2189

42. Komatsu Y, Majima T. Negative correlation between BMD and HbA1C in patients with type 2 diabetes. Clin Calcium. 2006;16 (8):1327-1331.

43. Rabijewski M, Papierska L, Piątkiewicz P. An association between bone mineral density and anabolic hormones in middle-aged and elderly men with prediabetes. Aging Male. 2017;20(3):205-213. doi:10.1080/13685538.2017.1338254

44. Gu L, Lai X, Wang Y, Zhang J, Liu J. A community-based study of the relationship between calcaneal bone mineral density and systemic parameters of blood glucose and lipids. Medicine. 2019;98(27): e16096. doi:10.1097/MD.0000000000016096
International Journal of General Medicine

\section{Publish your work in this journal}

The International Journal of General Medicine is an international, peer-reviewed open-access journal that focuses on general and internal medicine, pathogenesis, epidemiology, diagnosis, monitoring and treatment protocols. The journal is characterized by the rapid reporting of reviews, original research and clinical studies across all disease areas. The manuscript management system is completely online and includes a very quick and fair peer-review system, which is all easy to use. Visit http://www.dovepress.com/ testimonials.php to read real quotes from published authors. 